\title{
Estudo da relação anatômica do nervo sensitivo radial após fixação percutânea com fios de Kirschner*
}

\author{
Study of the anatomic relation of the radial sensory \\ nerve after percutaneous fixation with Kirschner wires
}

\author{
Pedro José Labronicl ${ }^{1}$, José Sergio Franco ${ }^{2}$, Rolix Hoffmann ${ }^{3}$, Anselmo Fernandes da Silva ${ }^{3}$, \\ Marco Aurélio Rodrigues da Fonseca Passos ${ }^{4}$, Paulo Roberto Barbosa de Toledo lourenço ${ }^{5}$, \\ Hélio Jorge Alvachian Fernandes ${ }^{6}$, Fernando Baldy dos Reis ${ }^{7}$
}

\section{RESUMO}

Objetivo: Determinar o risco de lesão do nervo sensitivo radial após fixação percutânea na região distal do rádio em cadáveres. Métodos: Foram utilizados 24 membros superiores de 12 cadáveres esqueleticamente maduros, 10 do sexo masculino e dois do feminino, com média de idade estimada de 50 anos. Um fio de Kirschner foi introduzido no processo estilóide do rádio para determinar: a distância entre o fio e o nervo sensitivo radial, entre o fio e o ramo do nervo dorsal mais próximo, entre o fio e os tendões do primeiro túnel osteofibroso e, finalmente, o número de ramos do nervo sensitivo radial. Resultados: A distância do ponto de emergência do nervo sensitivo radial, entre os tendões e o fio de Kirschner aplicado no processo do rádio,

* Trabalho realizado na Faculdade de Medicina de Petrópolis e no Serviço de Ortopedia e Traumatologia Prof. Dr. Donato D’ Ângelo, Hospital Santa Teresa, Petrópolis (RJ), Brasil.

1. Doutor em Medicina pela Universidade Federal de São Paulo Escola Paulista de Medicina e Chefe de Clínica do Serviço de Ortopedia e Traumatologia Prof. Dr. Donato D’Ângelo - Hospital Santa Teresa, Petrópolis (RJ), Brasil.

2. Chefe do Departamento e Professor Associado do Departamento de Ortopedia e Traumatologia da Faculdade de Medicina da Universidade Federal do Rio de Janeiro - UFRJ - Rio de Janeiro (RJ), Brasil.

3. Residente em Ortopedia e Traumatologia do Serviço de Ortopedia e Traumatologia Prof. Dr. Donato D’Ângelo - Hospital Santa Teresa, Petrópolis (RJ), Brasil. foi em média de $5,09 \mathrm{~cm}$, com desvio-padrão de $0,75 \mathrm{~cm}$. A distância entre o fio de Kirschner e o primeiro ramo dorsal foi em média de $4,33 \mathrm{~cm}$, variando entre zero e $10 \mathrm{~cm}$. $O$ número de ramos nervosos foi em média de quatro, variando entre dois a oito ramos. Conclusão: Os resultados deste estudo experimental indicaram que, quando o fio é introduzido no processo estilóide do rádio, o risco de lesão do nervo sensitivo radial é pequeno. Esse risco está diretamente relacionado com o número de ramos nervosos e a angulação dos fios durante sua introdução na extremidade distal do rádio.

Descritores - Fraturas do rádio/cirurgia; Fixação de fraturas/métodos; Fios ortopédicos; Procedimentos ortopédicos/métodos; Cadáver

4. Professor Titular de Anatomia da Faculdade de Medicina de Petrópolis; Professor Assistente de Anatomia e Médico da Universidade do Estado do Rio de Janeiro - UERJ - Rio de Janeiro (RJ), Brasil.

5. Médico Responsável pelo Grupo de Trauma do Hospital de Ipanema, Rio de Janeiro (RJ), Brasil.

6. Doutor, Professor Adjunto e Médico do Setor de Traumatologia do Aparelho Locomotor da Disciplina de Traumatologia do Departamento de Ortopedia e Traumatologia da Universidade Federal de São Paulo - UNIFESP - São Paulo (SP), Brasil.

7. Livre-Docente, Professor e Chefe do Setor de Traumatologia do Aparelho Locomotor da Disciplina de Traumatologia do Departamento de Ortopedia e Traumatologia da Universidade Federal de São Paulo - UNIFESP - São Paulo (SP), Brasil.

Endereço para correspondência: Av. Roberto Silveira, 187, apto. 601 - 25685-040 - Petrópolis (RJ), Brasil. Tel.: (24) $2242-5571$.

E-mail: plabronici@globo.com

Recebido em 14/8/07. Aprovado para publicação em 18/3/08.

Copyright RBO2008 


\section{ABSTRACT}

Objective: To establish the risk of lesion to the radial sensory nerve after percutaneous fixation in the distal radius region of cadavers. Methods: The authors used 24 upper limbs of 12 skeletally mature cadavers, 10 male, and 2 female, mean age estimated as 50 years. A Kirschner wire was introduced in the styloid process of the radius to make the following determinations: distance between the wire and the radial sensory nerve, between the wire and the closest dorsal nerve branch, between the wire and the tendons of the first osteofibrous tunnel, and, finally, the number of branches of the radial sensory nerve. Results: The site were the nerve appeared, between the two tendons and the Kirschner wire in the radial styloid process was a mean $5.09 \mathrm{~cm}$, with a standard deviation of $0.75 \mathrm{~cm}$. The distance between the Kirschner wire and the first dorsal branch was a mean $4.33 \mathrm{~cm}$, ranging from zero to $10 \mathrm{~cm}$. The number of nervous branches was a mean of four, ranging from two to eight branches. Conclusion: Results of this experimental study showed that when the wire is introduced in the styloid process of the radius, the risk of lesioning the radial sensory nerve is not a big risk. This risk is directly related to the number of nervous branches and to the angles of the wires while they are being introduced in the distal end of the radius.

\section{Keywords - Radius fractures/surgery; Fracture fixation/ methods; Bone wires; Orthopedic procedures/ methods; Cadaver}

\section{INTRODUÇÃO}

As fraturas da extremidade distal do rádio representam um sexto das que acometem os pacientes acima de 50 anos de idade ${ }^{(1)}$. Muitas dessas são fraturas estáveis que podem ser tratadas com redução incruenta e imobilização com aparelho gessado. Entretanto, fraturas instáveis e/ou com envolvimento da superfície articular podem comprometer o resultado final, se não forem tratadas adequadamente.

A osteossíntese com fios percutâneos tem sido utilizada nas fraturas da extremidade distal do rádio, tanto como tratamento único, quanto como complemento do fixador externo. É uma técnica excelente nas fraturas instáveis, intra e extra-articulares, e em combinação com outras técnicas ${ }^{(2-3)}$. Na literatura estão descritas várias formas de fixação percutânea aplicáveis à extremidade distal do rádio. Willenegger et al descreveram uma forma de realizar a redução incruenta e subseqüente colocação de dois fios de Kirschner de forma estática no processo estilóide, com os fios fixados na cortical oposta do rádio ${ }^{(4)}$. Fernandez et al e Trumble et al utilizaram o método de osteossíntese de Kapandji, no qual os fios eram inseridos na região dorsal, no traço da fratura (intrafocal) ${ }^{(5-6)}$. Fios percutâneos também podem ser utilizados, como joystick, na redução e fixação de pequenos fragmentos intraarticulares, conduzindo esses ossos para a posição de redução $0^{(7-9)}$.

O ramo sensitivo do nervo radial é conhecido por produzir dor quando lesado. Abrams et al observaram que esse ramo caminha sob o músculo braquiorradial, emerge entre este músculo e o extensor longo do carpo, permanece subcutâneo e corre em média por $9 \mathrm{~cm}$ ao longo e lateralmente ao processo estilóide ${ }^{(10)}$. Auerbach et al observaram entre três e 10 ramos distais do nervo sensitivo radial ${ }^{(11)}$. Devido às numerosas variações anatômicas, o nervo sensitivo radial nessa região está altamente suscetível a lesões durante a colocação de fios ou fixadores externos.

O objetivo deste trabalho foi determinar o risco de lesão do nervo sensitivo radial após fixação percutânea na região distal do rádio.

\section{MÉTODOS}

Foram utilizados 24 membros superiores de 12 cadáveres esqueleticamente maduros da Faculdade de Medicina de Petrópolis/RJ. Não foram evidenciadas deformidades prévias, fraturas ou cirurgias envolvendo os membros superiores. Dos cadáveres, 10 eram do sexo masculino e dois do feminino, com média de idade estimada de 50 anos. O posicionamento utilizado foi decúbito dorsal e não foram realizadas radiografias ou controle com fluoroscopia.

Após a localização do processo estilóide do rádio, por meio de palpação, este foi transfixado percutaneamente com um fio de Kirschner de 2,0mm, liso (Syn- 


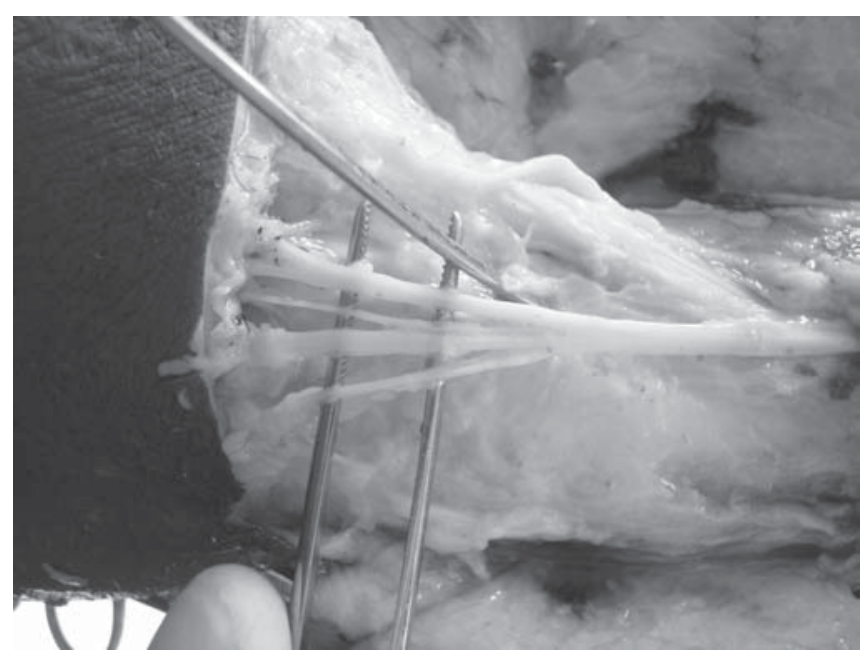

Figura 1 - Fio de Kirschner inserido no processo estilóide do rádio e os ramos do nervo sensitivo cutâneo radial

thes $\left.^{\circledR}\right)$. O pino foi introduzido sem incisão prévia da pele e de forma oblíqua, a $45^{\circ}$ em relação aos eixos coronal e sagital do rádio, avançando até a cortical oposta. Em seguida, a pele foi removida e o nervo sensitivo radial visibilizado. Esse procedimento foi realizado sempre pelo mesmo pesquisador. Não foi introduzido nenhum outro pino.

Os punhos foram dissecados, sem lupa, para determinar a distância entre o fio de Kirschner e o nervo sensitivo radial, a distância entre o fio e o ramo do nervo dorsal mais próximo, os tendões do primeiro túnel osteofibroso e o fio e o número de ramos do nervo (figura 1). Todas essas distâncias foram medidas com paquímetro.

Todos os fios foram removidos dos cadáveres ao final do experimento.

Este trabalho foi analisado e aprovado pelo Comitê de Ética da Faculdade de Medicina de Petrópolis/RJ.

A análise estatística foi realizada pelo teste dos postos sinalizados de Wilcoxon para verificar se existe diferença significativa entre o punho direito e esquerdo, ao nível de 5\%.

\section{RESULTADOS}

O ramo superficial do nervo radial foi localizado entre os tendões dos músculos braquiorradial e o extensor radial longo do carpo em todos os 24 antebraços. O local onde o nervo foi identificado, entre os dois tendões e o fio de Kirschner, na altura do processo estilóide do rádio, foi em média de $5,09 \mathrm{~cm}$, com desvio-padrão de $0,75 \mathrm{~cm}$. Não houve diferença significativa quando comparada a localização do nervo e o fio entre o antebraço direito com o esquerdo (tabelas 1 e 2).

Quando comparada a distância entre o fio de Kirschner, localizado no processo estilóide do rádio, e o primeiro ramo dorsal, encontramos média de $4,33 \mathrm{~cm}$, variando entre zero e $10 \mathrm{~cm}$. Também não houve dife-

TABELA 1

Análise entre as variantes pesquisadas no total da amostra

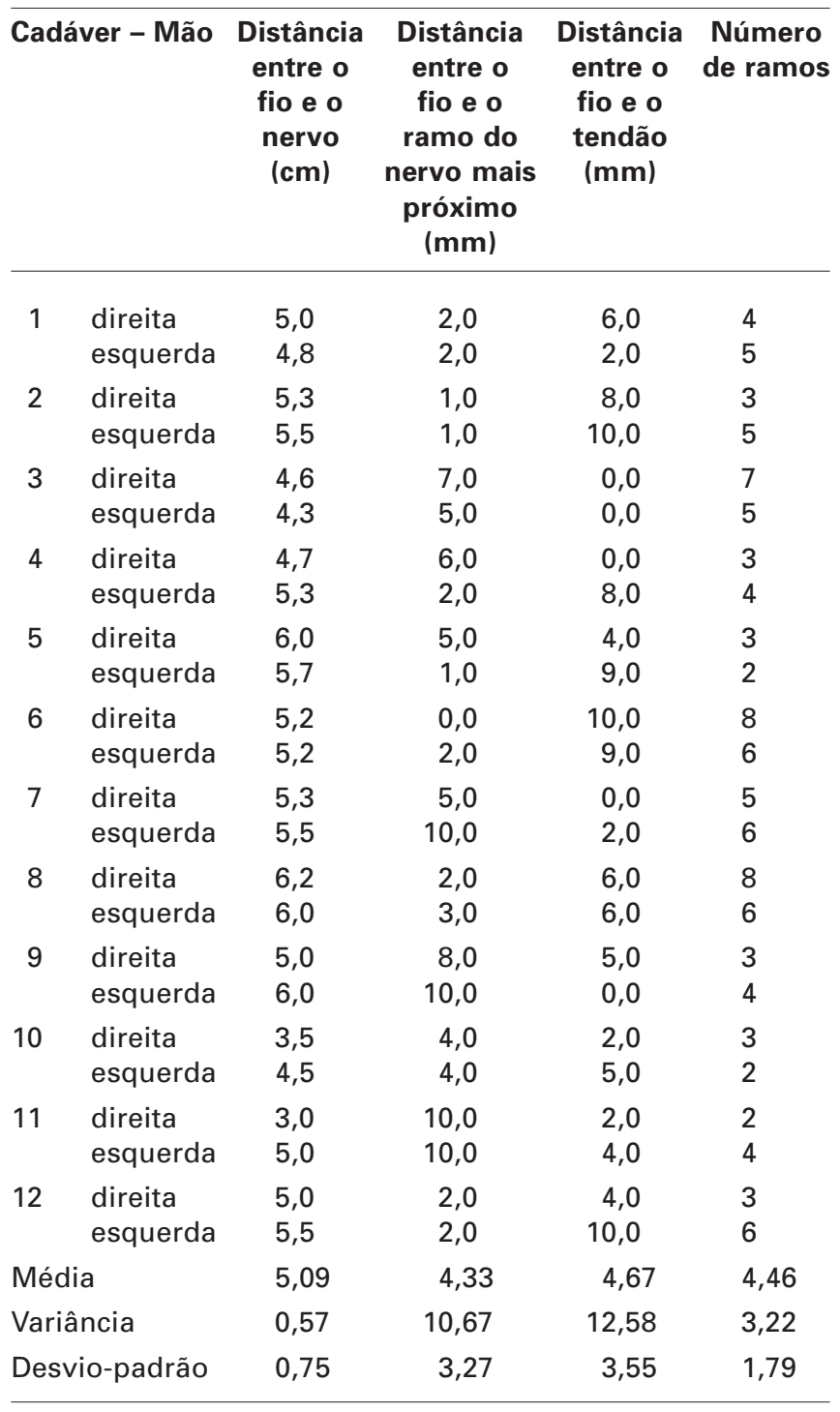

Fonte: Faculdade de Medicina de Petrópolis, Rio de Janeiro. 
TABELA 2

Análise estatística entre as variantes pesquisadas

\begin{tabular}{|c|c|c|c|c|c|c|c|c|}
\hline Cadáver & $\begin{array}{c}\text { Distância } \\
\text { fio-nervo } \\
\text { dir (cm) }\end{array}$ & $\begin{array}{l}\text { Distância } \\
\text { fio-ramo } \\
\text { dir (mm) }\end{array}$ & $\begin{array}{l}\text { Distância } \\
\text { fio-tendão } \\
\text { dir }(\mathrm{mm})\end{array}$ & $\begin{array}{c}\text { № de } \\
\text { ramos } \\
\text { dir }\end{array}$ & $\begin{array}{l}\text { Distância } \\
\text { fio-nervo } \\
\text { esq (cm) }\end{array}$ & $\begin{array}{l}\text { Distância } \\
\text { fio-ramo } \\
\text { esq ( } \mathrm{mm})\end{array}$ & $\begin{array}{l}\text { Distância } \\
\text { fio-tendão } \\
\text { esq (mm) }\end{array}$ & $\begin{array}{c}\text { № de } \\
\text { ramos } \\
\text { esq }\end{array}$ \\
\hline 1 & 5 & 2 & 6 & 4 & 4,8 & 2 & 2 & 5 \\
\hline 2 & 5,3 & 1 & 8 & 3 & 5,5 & 1 & 10 & 5 \\
\hline 3 & 4,6 & 7 & 0 & 7 & 4,3 & 5 & 0 & 5 \\
\hline 4 & 4,7 & 6 & 0 & 3 & 5,3 & 2 & 8 & 4 \\
\hline 5 & 6 & 5 & 4 & 3 & 5,7 & 1 & 9 & 2 \\
\hline 6 & 5,2 & 0 & 10 & 8 & 5,2 & 2 & 9 & 6 \\
\hline 7 & 5,3 & 5 & 0 & 5 & 5,5 & 10 & 2 & 6 \\
\hline 8 & 6,2 & 2 & 6 & 8 & 6 & 3 & 6 & 6 \\
\hline 9 & 5 & 8 & 5 & 3 & 6 & 10 & 0 & 4 \\
\hline 10 & 3,5 & 4 & 2 & 3 & 4,5 & 4 & 5 & 2 \\
\hline 11 & 3 & 10 & 2 & 2 & 5 & 10 & 4 & 4 \\
\hline 12 & 5 & 2 & 4 & 3 & 5,5 & 2 & 10 & 6 \\
\hline Média & 4,90 & 4,33 & 3,92 & 4,33 & 5,28 & 4,33 & 5,42 & 4,58 \\
\hline Desvio-padrão & 0,91 & 3,06 & 3,26 & 2,15 & 0,54 & 3,60 & 3,80 & 1,44 \\
\hline Mediana & 5,0 & 4,5 & 4,0 & 3,0 & 5,4 & 2,5 & 5,5 & 5,0 \\
\hline Mínimo & 3,0 & 0,0 & 0,0 & 2,0 & 4,3 & 1,0 & 0,0 & 2,0 \\
\hline Máximo & 6,2 & 10,0 & 10,0 & 8,0 & 6,0 & 10,0 & 10,0 & 6,0 \\
\hline$p$ valor $=$ & 0,13 & 1 & 0,18 & 0,68 & & & & \\
\hline
\end{tabular}

Fonte: Faculdade de Medicina de Petrópolis, Rio de Janeiro.

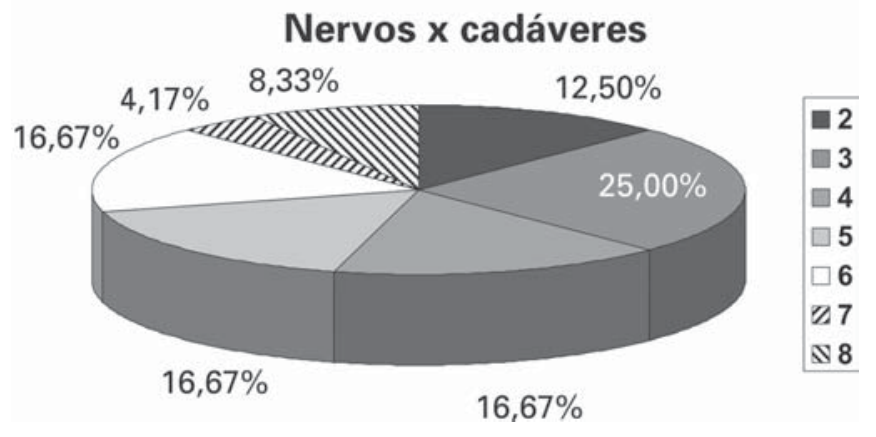

Gráfico 1 - Número de ramos da amostra

Fonte: Faculdade de Medicina de Petrópolis, Rio de Janeiro.

rença significativa quando comparada a distância do nervo e o fio entre o antebraço direito com o esquerdo (tabelas 1 e 2). O fio lesou o ramo dorsal do nervo em um cadáver, no punho à direita; por isso, a distância foi considerada zero.

Encontramos média de quatro ramos do nervo na região do rádio distal, variando entre dois e oito ramos (gráfico 1).

A distância entre o fio de Kirschner localizado no processo estilóide do rádio e os tendões do primeiro túnel osteofibroso foi em média de $4,67 \mathrm{~cm}$, variando entre zero e $10 \mathrm{~cm}$. Não houve diferença significativa entre o antebraço direito e o esquerdo (tabelas 1 e 2). O fio lesou os tendões do túnel osteofibroso em três cadáveres: um no punho direito, outro no punho esquerdo e, em um terceiro, em ambos os punhos; por isso a distância foi considerada zero.

\section{DISCUSSÃO}

Muitos procedimentos cirúrgicos são realizados sobre a região distal do rádio. A doença de De Quervain, sinovectomia e cisto artrossinovial do punho, laceração ou ruptura de tendão extensor, artroscopia de punho, instabilidade e fratura do carpo, artrites e fraturas do rádio distal são algumas das afecções que necessitam de atuação nesta área ${ }^{(11)}$.

A fixação percutânea com fios de Kirschner pode ser realizada como tratamento primário nas fraturas distais do rádio ou como complemento da fixação externa. É considerada excelente técnica nas fraturas instáveis, tanto extra como intra-articulares, principalmen- 
te nas fraturas dos tipos A2, A3, B1 e algumas do tipo C da classificação AO, porém, está contra-indicada nas fraturas gravemente cominuídas ou osteoporóticas, devido ao osso trabecular da região metafisária apresentar pouca estabilidade ${ }^{(2,12-14)}$.

A técnica de introdução de fios de Kirschner através do processo estilóide do rádio foi descrito primeiramente por Lambotte, em 1913, segundo Clancey ${ }^{(15)}$. Fritz et al relataram freqüentes aplicações da osteossíntese com fio de Kirschner em pacientes idosos ${ }^{(12)}$. Clancey relatou bons resultados após osteossíntese com fios de Kirschner, mas seus melhores resultados foram em pacientes jovens ${ }^{(15)}$.

Willenegger et al descreveram uma técnica em que, após a redução incruenta, introduzem dois fios de Kirschner no processo estilóide do rádio, de forma estática, ancorado na cortical oposta ${ }^{(4)}$.

Outra alternativa foi descrita por Kapandji e denominada de fixação intrafocal ${ }^{(16)}$. Nessa técnica, os fios de Kirschner são inseridos percutaneamente no foco da fratura, produzindo uma alavanca e realinhando o fragmento distal. Essa técnica é indicada para pacientes jovens com pouca cominuição e para os que são tratados menos de sete a 10 dias após o trauma. A vantagem da técnica intrafocal é que cria uma osteossíntese dinâmica, ao contrário da fixação precária estática conseguida pela técnica de Willenegger ${ }^{(16-18)}$.

A fixação com fios percutâneos também pode ser utilizada na redução e fixação de pequenos fragmentos intra-articulares. Nas fraturas do tipo C da classificação AO, com vários fragmentos intra-articulares, fios de Kirschner podem ser usados como um joystick para conduzir fragmentos ósseos na posição de redução( ${ }^{(2)}$.

Lesões do ramo sensitivo do nervo radial por incisões cirúrgicas são bem conhecidas e documentadas na literatura ${ }^{(19-24)}$. Após a fixação percutânea com fios, lesão do ramo sensitivo tem sido relatada ${ }^{(25-26)}$. Clinicamente, é clássico o desenvolvimento de parestesia, hiperestesia e neuromas dolorosos no dorso do punho e mão. Abrams et al examinaram 20 cadáveres e observaram que o ramo superficial do nervo radial se tornava subcutâneo em uma média de $9 \mathrm{~cm}$ proximal do processo estilóide do rádio (variando entre $7 \mathrm{~cm} \mathrm{e}$ $10,8 \mathrm{~cm})^{(10)}$. O ramo sensitivo do radial passa a distri- buir ramos dorsais, em média $5 \mathrm{~cm}$ proximais ao processo estilóide (variando entre $3,2 \mathrm{~cm}$ e $7,1 \mathrm{~cm}$ ).

Nosso estudo demonstrou que, após a fixação com fio de Kirschner introduzido no processo estilóide do rádio, a distância ficou em média em $5,0 \mathrm{~cm}$, variando entre $3,0 \mathrm{~cm}$ e $6,2 \mathrm{~cm}$, o que permite boa área de segurança para sua inserção. Quando observada a relação entre o fio e o primeiro ramo dorsal, a média da distância diminuiu para $4,3 \mathrm{~cm}$, em média, variando entre zero e $10 \mathrm{~cm}$. Apesar de a dissecção ter sido feita sem lupa, o número de ramos nervosos variou em média de quatro. Nossos resultados demonstraram que a fixação distal do rádio pode produzir lesão de ramos nervosos e está diretamente relacionada com o número de ramos.

Khawar et al analisaram a osteossíntese com fio percutâneo nas fraturas distais do rádio, em relação a deixar ou não o fio externo. Os fios, quando cobertos, previnem infecções no ponto de entrada e aumentam o conforto e a satisfação dos pacientes. Entretanto, não encontraram diferença entre os grupos em relação à satisfação, dor durante o período de fixação, lesão do nervo cutâneo radial ou infecção no local dos fios(26).

Lenoble et al relataram que as lesões do nervo cutâneo radial foram mais comuns após a cirurgia de Kapandji. As complicações estavam mais relacionadas com a remoção do fio do que com a movimentação precoce do punho. Relataram que, no ato da retirada do fio, o nervo pode estar envolvido pelo tecido cicatricial e desenvolver a lesão e, por isso, aconselham dissecção do nervo. Também notaram que, na fixação do processo estilóide do rádio, desenvolveram menor incidência de lesão nervosa, pois os fios se localizam mais distais, quando comparada com a colocação do fixador externo ${ }^{(27)}$.

\section{CONCLUSÃO}

Os resultados deste estudo experimental indicaram que, quando o fio é introduzido no processo estilóide do rádio, o risco de lesão do nervo sensitivo radial é pequeno. Esse risco está diretamente relacionado com o número de ramos nervosos e a angulação dos fios durante sua introdução na extremidade distal do rádio. 


\section{REFERÊNCIAS}

1. Jupiter JB. Fractures of the distal end of the radius. J Bone Joint Surg Am. 1991;73(3):461-9.

2. Simic PM, Weiland AJ. Fractures of the distal aspect of the radius: changes in treatment over the past two decades. Instr Course Lect. 2003;52:185-95.

3. Strohm PC, Müller CA, Boll T, Pfister U. Two procedures for Kirschner wire osteosynthesis of distal radial fractures. A randomized trial. J Bone Joint Surg Am. 2004;86-A(12):26218.

4. Willenegger H, Guggenbühl A. [Operative treatment of certain cases of distal radius fracture]. Helv Chir Acta. 1959;26(2):8194. German.

5. Fernandez DL, Palmer AK. Fractures of the distal radius. In: Green DP, Hotchkiss RN, Pederson WC, editors. Green's operative hand surgery. 4th ed. New York: Churchill Livingstone; 1999. p. 929-85.

6. Trumble TE, Wagner W, Hanel DP, Vedder NB, Gilbert M. Intrafocal (Kapandji) pinning of distal radius fractures with and without external fixation. J Hand Surg [Am]. 1998;23(3):38194. Comment in: J Hand Surg [Am]. 1998;23(6):1119-21.

7. Jupiter JB, Fernandez DL, Whipple TL, Richards RR. Intraarticular fractures of the distal radius: contemporary perspectives. Instr Course Lect. 1998;47:191-202.

8. Duncan SF, Weiland AJ. Minimally invasive reduction and osteosynthesis of articular fractures of the distal radius. Injury. 2001;32 Suppl 1:SA14-24.

9. Geissler WB, Fernandez DL. Percutaneous and limited open reduction of the articular surface of the distal radius. J Orthop Trauma. 1991;5(3):255-64.

10. Abrams RA, Brown RA, Botte MJ. The superficial branch of the radial nerve: an anatomic study with surgical implications. $J$ Hand Surg [Am]. 1992;17(6):1037-41.

11. Auerbach DM, Collins ED, Kunkle KL, Monsanto EH. The radial sensory nerve. An anatomic study. Clin Orthop Relat Res. 1994;(308):241-9.

12. Fritz T, Wersching D, Klavora R, Krieglstein C, Friedl W. Combined Kirschner wire fixation in the treatment of Colles fracture. A prospective, controlled trial. Arch Orthop Trauma Surg. 1999;119(3-4):171-8.

13. Hermichen HG, Hansis M. [Bore wire osteosynthesis in distal radius fractures]. Aktuelle Traumatol. 1987;17(3):109-12. German.
14. Letsch R, Schmit-Neuerburg KP, Schax M. [Choice of surgical procedure of the distal radius. Bore wire versus plate. Aktuelle Traumatol. 1987;17(3):113-9. German.

15. Clancey GJ. Percutaneous Kirschner-wire fixation of Colles fractures. A prospective study of thirty cases. J Bone Joint Surg Am. 1984;66(7):1008-14.

16. Kapandji A. [Internal fixation by double intrafocal plate. Functional treatment of non articular fractures of the lower end of the radius (author's transl)]. Ann Chir. 1976;30(11-12):9038. French.

17. Fritz T, Heyer T, Krieglstein C, Mattern R, Kallieris D, Friedl W. [Biomechanics of combined Kirschner wire osteosynthesis in the human model of unstable dorsal, distal radius fractures (Colles type)]. Chirurg. 1997;68(5):496-502. German.

18. Nonnenmacher J, Soley K, Bahm J. [Intrafocal wire fixation of wrist fractures. The original Kapandji technique. Course. Review of 400 cases]. Chirurgie. 1994-1995;120(3):119-27. French.

19. Arons MS. de Quervain's release in working women: a report of failures, complications, and associated diagnoses. J Hand Surg [Am]. 1987;12(4):540-4.

20. Braidwood AS. Superficial radial neuropathy. J Bone Joint Surg Br. 1975;57(3):380-3.

21. Dellon AL, Mackinnon SE. Susceptibility of the superficial sensory branch of the radial nerve to form painful neuromas. J Hand Surg [Br]. 1984;9(1):42-5.

22. Griffiths JC. Nerve injuries after plating of the forearm bones. Br Med J. 1966;2(5508):277-9.

23. Linscheid RL. Injuries to radial nerve at wrist. Arch Surg. 1965;91(6):942-6.

24. Fernández DL. Fractures of the distal radius: operative treatment. Inst Course Lect. 1993;42:73-88. Review.

25. Rayhack JM. The history and evolution of percutaneous pinning of displaced distal radius fractures. Orthop Clin North Am. 1993;24(2):287-300.

26. Khawar W, Mulhall K, Mwaura B, Kaar K. Percutaneous wire fixation of distal radial fractures: Is it preferable to bury the wires? J Bone Joint Surg Br. 2004;86-B(Suppl 2):125-6.

27. Lenoble E, Dumontier C, Goutallier D, Apoil A. Fracture of the distal radius. A prospective comparison between trans-styloid and Kapandji fixations. J Bone Joint Surg Br. 1995;77(4):5627. 